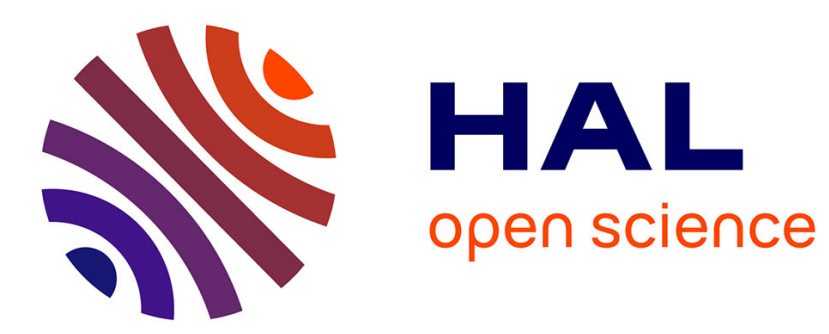

\title{
General Relativistic Flux Modulations from Disk Instabilities in Sagittarius A
}

\author{
M. Falanga, F. Melia, Michel Tagger, A. Goldwurm, G. Belanger
}

\section{To cite this version:}

M. Falanga, F. Melia, Michel Tagger, A. Goldwurm, G. Belanger. General Relativistic Flux Modulations from Disk Instabilities in Sagittarius A. The Astrophysical journal letters, 2007, 662, pp.L15-L18. 10.1086/519278 . in2p3-00145578

\section{HAL Id: in2p3-00145578 https://hal.in2p3.fr/in2p3-00145578}

Submitted on 1 Jul 2015

HAL is a multi-disciplinary open access archive for the deposit and dissemination of scientific research documents, whether they are published or not. The documents may come from teaching and research institutions in France or abroad, or from public or private research centers.
L'archive ouverte pluridisciplinaire HAL, est destinée au dépôt et à la diffusion de documents scientifiques de niveau recherche, publiés ou non, émanant des établissements d'enseignement et de recherche français ou étrangers, des laboratoires publics ou privés. 
Draft VERSION November 19, 2013

Preprint typeset using LTEX style emulateapj v. 08/22/09

\title{
GENERAL RELATIVISTIC FLUX MODULATIONS FROM DISK INSTABILITIES IN SAGITTARIUS A*
}

\author{
Maurizio Falanga, ${ }^{1,2}$ Fulvio Melia, ${ }^{3,4}$ Michel Tagger, ${ }^{1,5}$ \\ Andrea Goldwurm, ${ }^{1,5}$ and Guillaume Bélanger ${ }^{6}$ \\ Draft version November 19, 2013
}

\begin{abstract}
Near-IR and X-ray flares have been detected from the supermassive black hole Sgr A* at the center of our Galaxy with a (quasi)-period of $\sim 17-20$ minutes, suggesting an emission region only a few Schwarzschild radii above the event horizon. The latest X-ray flare, detected with XMM-Newton, is notable for its detailed lightcurve, yielding not only the highest quality period thus far, but also important structure reflecting the geometry of the emitting region. Recent MHD simulations of Sgr A*'s disk have demonstrated the growth of a Rossby wave instability, that enhances the accretion rate for several hours, possibly accounting for the observed flares. In this Letter, we carry out ray-tracing calculations in a Schwarzschild metric to determine as accurately as possible the lightcurve produced by general relativistic effects during such a disruption. We find that the Rossby wave induced spiral pattern in the disk is an excellent fit to the data, implying a disk inclination angle of $\approx 77^{\circ}$. Note, however, that if this association is correct, the observed period is not due to the underlying Keplerian motion but, rather, to the pattern speed. The favorable comparison between the observed and simulated lightcurves provides important additional evidence that the flares are produced in Sgr A*'s inner disk.
\end{abstract}

Subject headings: accretion—black hole_Galaxy: center—instabilities—magnetohydrodynamics—relativity

\section{INTRODUCTION}

Long-term monitoring of $\mathrm{Sgr} \mathrm{A}^{*}$ has thus far resulted in the discovery of several near-IR (Genzel et al. 2003) and Xray flares (Baganoff et al. 2001) from this object. One of the most recent events, observed with XMM-Newton in 2004, has produced compelling evidence of a significant modulation in the X-ray lightcurve with a quasi-period $\sim 22.2$ minutes (Bélanger et al. 2006). Similar periodic fluctuations had also been seen earlier in the near-IR events.

A period of $\sim 22.2$ minutes is rather intriguing because simple considerations (Melia 2001) would place the corresponding emission region at roughly 3 Schwarzschild radii $\left(r_{s} \equiv 2 G M / c^{2}\right)$ for a black hole $(\mathrm{BH})$ mass of $\sim 3.4 \times 10^{6}$ $\mathbf{M}_{\odot}$ (Schödel et al. 2003). It is not yet understood, however, why the Keplerian radius corresponding to this period is actually less than $3 r_{s}$. It could be that the difference is due to the spin of the $\mathrm{BH}$, which moves the inner disk radius inwards for prograde rotation. Or it could be that the so-called stress edge - the location where the inspiralling material actually detaches from the rest of the magnetized disk-is dynamically important in establishing where the disk emission terminates (Krolik \& Hawley 2002; Melia et al. 2007).

Part of the uncertainty is due to the fact that the structure of the disk surrounding $\mathrm{Sgr} \mathrm{A}^{*}$ is itself not fully understood yet. We have recently begun to simulate the behavior of the hot, magnetized disk during a disruptive event that may be responsible for the flares, under the assumption that the instability is induced by low angular momen-

\footnotetext{
${ }^{1}$ CEA Saclay, DSM/DAPNIA/Service d'Astrophysique, 91191 Gif-surYvette, France; mfalanga@cea.fr

${ }^{2}$ AIM - Unité Mixte de Recherche CEA - CNRS - Université Paris 7 . UMR n 7158

${ }^{3}$ Physics Department and Steward Observatory, The University of Arizona, Tucson, AZ 85721

${ }^{4}$ Sir Thomas Lyle Fellow and Miegunyah Fellow.

${ }^{5}$ Université Paris Diderot-Paris 7 et Observatoire de Paris, Laboratoire APC, Paris, France

${ }^{6}$ ESA/ESAC, Apartado 50727, 28080 Madrid, Spain.
}

tum clumps of plasma "raining" inwards towards the Keplerian region (Tagger \& Melia 2006; see also Chan et al. 2006). This scenario is motivated by extensive hydrodynamic (Melia \& Coker 1999; Cuadra et al. 2005) and MHD (Igumenshchev \& Narayan 2002) simulations which show that for the stellar-wind fed conditions at the Galactic center, the average specific angular momentum of gas captured gravitationally by $\mathrm{Sgr}$ A* is too small to sustain a 'conventional' (i.e., typically large $\sim 10^{5} r_{s}$ ) disk. Instead, only clumps of plasma with relatively small angular momentum venture inwards and merge with-essentially, 'rain' onto- the compact disk at the circularization radius, which for $\operatorname{Sgr} \mathrm{A}^{*}$ is $\lesssim 10-10^{3} r_{s}$ (see also Melia \& Falcke 2001; Melia 2007).

These MHD simulations (Tagger \& Melia 2006) did indeed establish the result that the merger of an infalling blob with the existing Keplerian flow induces a Rossby wave instability (RWI) leading to the total disruption of the disk on a timescale relevant to the Sgr A* flare phenomenon. But unless the ensuing X-ray modulation is due to a QPO response in the inspiralling matter (see Chan et al. 2006), one cannot escape the fact that general-relativistic effects are essential in producing a periodicity in the lightcurve due to radiation by an azimuthally asymmetric emitter. The pattern of modulation over one complete cycle would be the result of several influences, including a Doppler shift, light-bending, and lensing effect near the BH's event horizon (e.g., Hollywood et al. 1995).

In this Letter, we take the results from the (non-relativistic) MHD simulation of Tagger \& Melia (2006) and carry out a full ray-tracing calculation of the lightcurve produced by the disruption for direct comparison with the XMM-Newton data.

\section{OBSERVED LIGHTCURVE OF AN X-RAY FLARE FROM SGR A*}

An inspection of Fig. 5 in Bélanger et al. (2006) shows that the effects of gravitational light-bending, lensing, Doppler effect and travel time delay may have helped to shape the folded lightcurves from the 2004 August 31 event. One cannot yet discount the possibility that an actual periodic dynamical effect may have also contributed to the modulation seen in the 
emissivity, but this is necessarily model-dependent and the signature may not be unique. For example, Tagger \& Melia (2006) found that a quasi-periodic modulation could be excited by non-linearities in the evolution of the spiral-Rossby pattern, which would sit on top of the modulation one would see due to general relativistic effects. For the purposes of this Letter, we will adopt the simplest assumption-that the modulation is due predominantly to general relativistic effects.

The $2-10 \mathrm{keV}$ folded curve from the 2004 August 31 event (see Fig. 3) looks broader and, except for one significant datum standing well away from the rest, looks more like a continuous, relatively smooth modulation rather than the sharp changing profile produced by an orbiting hot spot (see e.g., Hollywood et al. 1995). It is partially for this (phenomenological) reason that the Rossby wave instability is promising and worth investigating further here, because the disruption it causes is global, offering the possibility of producing a gradual modulation in the lightcurve when general relativistic effects are included.

\section{NUMERICAL SIMULATION OF THE INSTABILITY}

The instability we have simulated with our MHD code has a long history, dating back to Lovelace \& Hohlfeld (1978), who showed that a disk presenting an extremum of a quantity $\mathcal{L}$ (later dubbed vortensity) was subject to a local instability of Rossby vortices. The requirement of an extremum is similar to that giving rise to the Kelvin-Helmholtz instability of sheared flows. More recently, Lovelace et al. (1999) renamed it the Rossby Wave Instability (RWI) and developed the theory and numerical simulation.

In isothermal, unmagnetized disks, $\mathcal{L}$ is the specific vorticity averaged across the disk thickness,

$$
\mathcal{L}=\frac{\vec{\nabla} \times \vec{V}}{\Sigma}=\frac{\kappa^{2}}{2 \Omega \Sigma},
$$

where $\Sigma$ is the disk's surface density, $\Omega$ its rotation frequency, and $\kappa^{2}=4 \Omega^{2}+2 \Omega \Omega^{\prime} r$ is the epicyclic frequency squared. The extremum of $\mathcal{L}$ appears to be due to an extremum in the radial density profile. To understand how the instability is driven, we note that Rossby waves in disks form normal modes trapped near the extremum of $\mathcal{L}$. In the MHD form of the RWI, the disk is threaded by a vertical (poloidal) magnetic field $B_{0}(r)$. Its properties are essentially the same as those discussed above, except that here the critical quantity is $\mathcal{L}_{B}=\kappa^{2} \Sigma /\left(2 \Omega B_{0}^{2}\right)$, and the growth rate can be higher because of the long-range action of the Lorentz force coupling the Rossby vortices.

\section{RAY TRACING CALCULATIONS}

A typical profile of the inner disk during the Rossby wave growth is shown in Fig. 3 of Tagger \& Melia (2006). In this paper, we present the lightcurve and images associated with this disruption, from the vantage point of an observer at infinity. The calculation is carried out with a fully general relativistic ray-tracing code. The RWI arises in the compact accretion disk surrounding the (Schwarzschild) $\mathrm{BH}$, and we describe its morphology using coordinates in the co-rotating frame $(r, \theta, \varphi)$. The modeled accretion disk is thin and the RWI may be considered to lie in the equatorial plane $(\theta=\pi / 2)$ of the compact object. The observer is located at infinity with viewing angle $i$ relative to the $z^{\prime}$-axis in the non-rotating frame, at (observer) polar coordinates $\left(r^{\prime}, \theta^{\prime}, \varphi^{\prime}\right)$. The deflection angle of a photon emitted by plasma in the Rossby-unstable region is $\psi$, and varies periodically with $\cos \psi=\cos i \cos \varphi$.
These emitted photons are deflected by the $\mathrm{BH}$ and intersect the observer's detector plane at infinity. The distance between the line-of-sight and the point at which the photon reaches the detector is defined as the impact parameter $b$. Using this geometry, the deflection angle of the photon's trajectory may be obtained with the light-bending relation between $\alpha$ (the angle between the emission direction of the photon and the direction from the center of the $\mathrm{BH}$ to the location of the emitter) and $\psi$ from the geodesic equation

$$
\psi=\int_{R}^{\infty} \frac{d r}{r^{2}}\left[\frac{1}{b^{2}}-\frac{1}{r^{2}}\left(1-\frac{r_{s}}{r}\right)\right] .
$$

This procedure yields the impact parameter $b=r(1-$ $\left.r_{s} / r\right)^{-1} \sin \alpha$ of the photons in terms of the emitting radius $r$, and ultimately allows us to calculate the flux at infinity. A detailed description of this geometry is provided in, e.g., Luminet (1979), and Falanga et al. (2007). In our derivation, we shall use the same notation and geometry described in Poutanen \& Gierliński (2003), though pertaining to a localized region (or clump) in the disk. The system of units is chosen such that $G=c=1$; in this standard coordinate system, the BH's horizon occurs at the Schwarzschild radius $r_{s}=2 M$, where $M$ is the mass of the compact object.

In the simulations we report here, the emitting region is geometrically thin, and generally optically thin as well. Our rays include an integration through the whole emitting depth, but because most of the emissivity is concentrated near the disk's plane, we effectively have a situation in which the rays themselves appear to begin very close to this plane. Rays leaving the disk in directions that eventually take them around the $\mathrm{BH}$, heading toward the observer, contribute much less to the overall flux and we ignore them here.

The general-relativistic effects to be considered are now: (i) light-bending (see above), (ii) gravitational Doppler effect defined as $(1+\mathrm{z})$, (iii) gravitational lensing effect, $d \Omega_{\mathrm{obs}}=$ $b d b d \varphi / D^{2}$ (with $D$ the distance to the source), expressed through the impact parameter, and (iv) the travel time delay. We calculate the relative time delay between photons arriving at the observer from different parts of the disk, using the geodesic equation. The first photon reaching the observer is the photon emitted at phase $\varphi=0$, on the closest radius $\left(r=r_{\text {max }}\right)$ to the observer. We set this reference time, $T_{0}$, equal to zero.

The observed flux at energy $E^{\prime}$ is $F_{\text {obs }}\left(E^{\prime}\right)=I_{\text {obs }}\left(E^{\prime}\right) d \Omega_{\text {obs }}$, where $I_{\mathrm{obs}}\left(E^{\prime}\right)$ is the radiation intensity observed at infinity and $d \Omega_{\mathrm{obs}}$ is the solid angle on the observer's sky including relativistic effects. Using the relation $I_{\mathrm{obs}}\left(E^{\prime}, \alpha^{\prime}\right)=$ $(1+z)^{-3} I_{\mathrm{em}}(E, \alpha)$, a Lorentz invariant quantity that is constant along null geodesics in vacuum, the intensity of a light source integrated over its effective energy range is proportional to the fourth power of the redshift factor, $I_{\mathrm{obs}}\left(\alpha^{\prime}\right)=(1+z)^{-4} I_{\mathrm{em}}(r, \varphi)$, $I_{\mathrm{em}}(r, \varphi)$ being the intensity measured in the rest frame of the clump (Misner, Thorne, \& Wheeler 1973).

Although our magnetohydrodynamic simulation, and corresponding ray-tracing calculation, are quite sophisticated, they are nonetheless still somewhat restricted in that we have not allowed for a completely self-consistent coupling between the plasma and the radiation. The infalling plasma radiates inefficiently, so this is not a serious deficiency as far as the dynamics is concerned. Furthermore, properly modelling the compression of the gas would require fully 3-D simulations, which MHD codes are still unable to handle in the conditions (a disk threaded by a near-equipartition vertical field) that we 


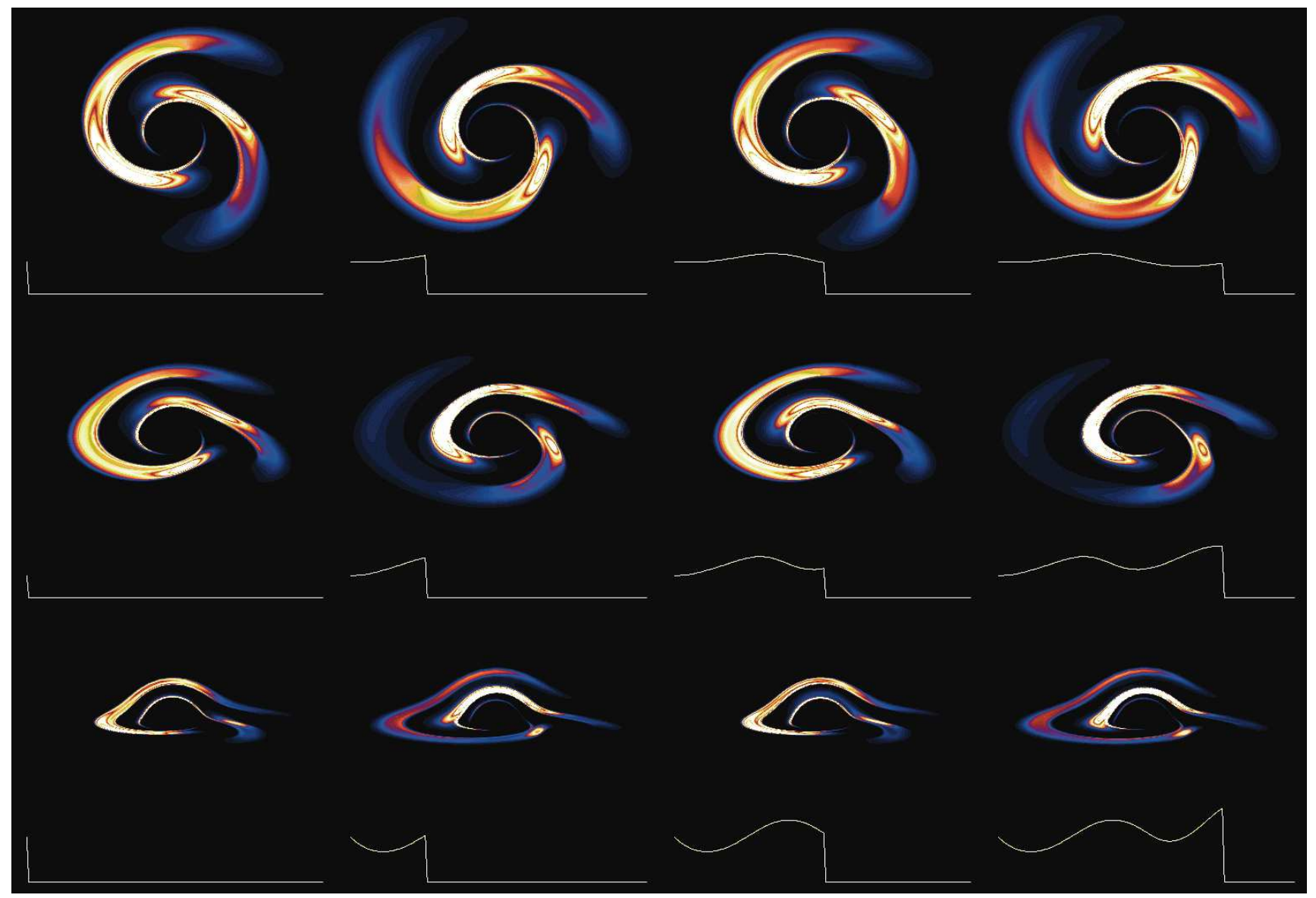

FIG. 1. - Horizontal: Four snapshots of the disk (spiral pattern) as it would appear to an observer looking along a line-of-sight inclined by $30^{\circ}$ (top), $60^{\circ}$ (middle), and $80^{\circ}$ (bottom), relative to the disk's symmetry axis, at phases $\varphi=0, \pi / 2, \pi$, and $3 \pi / 2$, respectively. Variation in each column is due to general relativistic light-bending, lensing, and Doppler shifts, including the relativistic time delay for the various inclinations. The lightcurves attached to their respective images show how an observer views these overall effects from infinity (see also Fig. 2). Slight differences between the two arms, resulting from the MHD simulations, result in two slightly different peaks in the lightcurve during one full rotation of the pattern.

use. For simplicity, the MHD simulation was thus carried out assuming isothermal conditions. However the electron temperature, which dominates the radiative emission, responds to the compression of the gas and we model this here with an ad-hoc prescription, from the compression obtained in the simulation. This is sufficient to provide the main result of the present work, which is the form of the light curve that the spiral pattern and general relativistic effects generate. Also, we have not included non-local effects arising, e.g., from inverse Compton scattering. In determining the surface emissivity, it therefore makes sense to take a simplified approach in which we include principally the parameter scalings, rather than their absolute values. This procedure will give us correct amplitudes in the lightcurve, though not the absolute value of the flux per se.

Using the perfect gas law for an adiabatic flow (since the gas is radiatively inefficient), we can invoke a polytropic equation of state with $\gamma=5 / 3$ and write the temperature as $T \propto \rho^{2 / 3}$. This assumes further that the radiation pressure is negligible and $T$ is not so high that the particles are strongly relativistic. Although the disk is not in full hydrostatic equilibrium, we can still argue that on average, we should have for a steady thin disk the gas density $\rho=\Sigma / H(r, z)$, where $\Sigma$ is the column density, and $H$ is the disk height. These give $\rho \propto \Sigma^{3 / 4} r^{-9 / 8}$ (using $r$ to denote the radius in the equatorial plane) and $T \propto \Sigma^{1 / 2} r^{-3 / 4}$. The synchrotron emissivity is therefore $j_{s} \propto B n_{\mathrm{nt}} \propto \Sigma(T \rho) \propto \Sigma \rho^{(5 / 3)}$, where the nonthermal par- ticle energy is roughly in equipartition with the thermal. We argue that the plasma is fully ionized and resistivity is minimal, so $B$ is frozen into the gas, which means that $B \propto \Sigma$. We therefore infer that $j_{s} \propto \Sigma^{9 / 4} r^{-15 / 8}$.

Now the X-rays are produced via inverse Compton scattering from the seed photon number flux. Thus, with $L_{\text {seed }} \propto$ $r^{3} j_{s}$, where $j_{s}$ is the synchrotron emissivity in units of energy per unit volume per unit time, the soft photon flux scales as the emitted power divided by the characteristic area. That is, $F_{\text {seed }} \propto r^{3} j_{s} / r^{2}=r j_{s}$, which is going to be roughly the same scaling as the seed photon density, so $n_{\text {seed }} \propto r j_{s} \propto \Sigma^{9 / 4} r^{-7 / 8}$. The inverse Compton scattering emissivity is therefore $j_{i c} \propto$ $n_{\mathrm{nt}} n_{\text {seed }} \propto \Sigma^{7 / 2} r^{-11 / 4}$. Thus, $j_{X-\text { ray }} \sim j_{i c}$, and the surface intensity is $I_{\mathrm{em}} \propto \int j_{X-r a y} d s \propto j_{X-\text { ray }} H$, which gives finally $I_{\mathrm{em}} \propto \Sigma^{15 / 4} r^{-13 / 8}$. The core physics in this expression is the surface density $\Sigma(r, \varphi)$, which we take from the MHD simulation in Tagger \& Melia (2006).

In order to evaluate the flux at a given azimuthal angle $\varphi$ and radius $r$, we first compute numerically $\psi(\alpha)$, and then calculate the Dopper shift, lensing effects, and finally the flux $F_{\text {obs }}$ as a function of the arrival time, including all the travel time delays described above. The simulation is carried out on a polar grid with $n_{r}=256$ and $n_{\varphi}=128$ points, extending from $r_{\text {in }}=3 r_{s}$ to $90 r_{s}$. In this pass through the problem, in order to clearly separate the general relativistic modulation from the complex dynamical behaviour observed during the simulation, we have chosen to take a representative snapshot 
and rotate it at the frequency measured in the simulation. A full treatment of the whole simulation will be presented in Falanga et al. (2007).

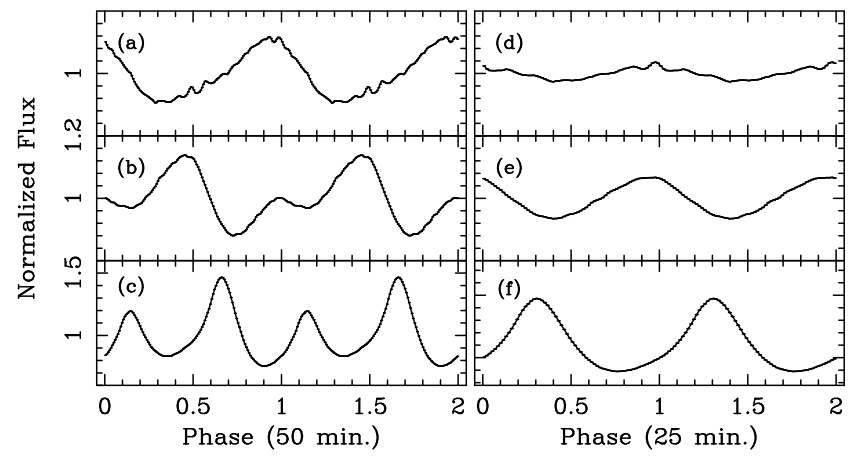

FIG. 2.- Panels (a-c) show the lightcurve observed at infinity, resulting from general relativistic flux modulations associated with a Rossby-wave-disrupted disk. The period arises from the two-spiral-armed pattern speed, and is roughly 50 minutes for this particular MHD simulation. Panel (a) is for a disk inclination of $30^{\circ}$, (b) is $60^{\circ}$, and (c) is $80^{\circ}$, in a one-to-one correspondence with the rows of Fig. 1. Panels (d-f) show the same lightcurve, though now folded on half of a pattern-rotation period, i.e., 25 minutes. Each period is repeted once for clarity.

\section{RESULTS AND DISCUSSION}

Our principal results are presented graphically below, and may be understood with reference to the simulated images shown in Fig. 11. These are intensity maps projected onto the plane of the sky, for the ray-traced perturbed disk in Tagger \& Melia (2006), at three different inclination angles: $30^{\circ}$ in the top row, $60^{\circ}$ in the middle, and $80^{\circ}$ for the bottom row. The four columns are snapshots taken at 4 (equallyspaced) phases of one complete pattern revolution. The general relativistic distortions depend strongly on inclination angle, which we employ in our search for the best fit to the modulation in the X-ray lightcurve.

This variation is demonstrated quantitatively in Fig. 2 which shows the lightcurves corresponding to the three inclinations illustrated in Fig. 1. Note that at small inclination, we detect a gradual, broad modulation, whereas for the higher inclinations, we begin to see the effects of a two-spiral arm emitting region. Interestingly, the period associated with the pattern rotation in the Tagger \& Melia (2006) simulation is about 50 minutes. Though this calculation was not optimized to fit the observed period, the fact that we see a bimodal modulation from the two spiral arms suggests that one cycle in the data may in fact correspond to half a revolution of the pattern. In this figure, we therefore also show the calculated lightcurve folded over half a pattern period, corresponding to about 25 minutes.

The true test of relevance for this simulation lies in a direct comparison between the data and the inclination-dependent lightcurves. We emphasized in the introduction that the various relativistic effects produce a unique profile, not easily confused with other periodic modulations, most of which tend to be sinusoidal. As we demonstrate in Fig. 3, the shape of the calculated lightcurve, particularly its amplitude, is quite sensitive to the inclination angle, which again, is most easily understood with reference to Fig. 1 For example, this figure includes 3 curves, corresponding to inclinations of $70^{\circ}, 77^{\circ}$, and $80^{\circ}$. The middle curve produces the best fit $\left(\chi_{\text {red }}^{2}=1.1\right)$, and it should be noted that the correspondence to the data is excellent, not only in terms of the modulation amplitude, but also for the shape of the lightcurve itself. Note that the simulated lightcurve corresponds to the bolometric flux emission. We have therefore compared the theoretical curve with the observed 2-10 keV lightcurve, rather than individually in different energy bands, as presented in Bélanger et al. (2006).

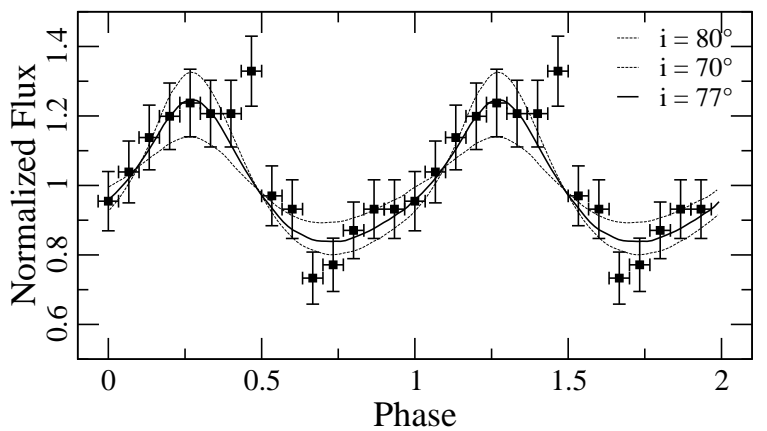

FIG. 3.- Lightcurve of the 2004, August 31 flare folded with a phase of $1330 \mathrm{~s}$, in the $2-10 \mathrm{keV}$ energy band from Bélanger et al. (2006). The best fit model is shown by the solid line using an inclination angle of $77^{\circ}$. The dasehed line represent upper and lower limits using $80^{\circ}$ (bigger amplitude) and $70^{\circ}$ (smaller amplitude) inclination angles, respectively.

There are several new ideas that we can take away from this work. First, if the periodic modulation seen in some flares from $\mathrm{Sgr} \mathrm{A}^{*}$ by both IR and X-ray instruments is real, it is not at all obvious that the periodicity is due to an underlying Keplerian period. One must be very careful, therefore, in over-interpreting these periods in terms of a BH spin. Second, the shape of the X-ray lightcurve in this particular flare is too broad for it to correspond easily to a highly localized hot spot on the disk, which instead would produce a more strongly peaked profile like that investigated earlier by Hollywood et al. (1995). Instead, our work here argues for a more global disruption in the disk, at least for some events, like the 2004, August 31 flare observed with XMM-Newton. In this regard, the driving mechanism is likely to be an infall of clumps of plasma that merge with the existing compact disk and induce a Rossby-type of instability. Finally, the observed power density spectrum shows that the (quasi)-period is quite clean, without any evidence that the emission region is spread over a large range in Keplerian periods. This has been taken as some evidence that the disturbance must therefore be highly localized, probably near the marginally stable orbit. But as we have shown here, the disturbance need not be that close to the event horizon in order to produce a modulation with a period of only $\sim 20-25$ minutes. One may still get a narrow peak in the power density spectrum, as long as the modulation is due to a pattern rotation, rather than to motion along an orbit. But this will only work as long as the pattern has multiple components, such as the two spiral arms we have modeled in this paper.

The research was partially supported by the French Space Agency (CNES) and NSF grant AST-0402502 at the University of Arizona. FM is grateful for the hospitality of the APC in Paris, where most of this work was carried out. 
Chan, C., Liu, S., Fryer, C. L., et al. 2006, ApJ, submitted astro-ph/0611269 Cuadra, J., Nayakshin, S., Springel, V. \& Di Matteo, T. 2005, MNRAS, 360, L55

Falanga et al., ApJ, 2007, in preparation

Genzel, R. et al. 2003, Nature, 425, 934

Hollywood, J. M., Melia, F., Close, L. M., et al. 1995, ApJ, 448, L21

Igumenshchev, I.V. \& Narayan, R. 2002, ApJ, 566, 137

Krolik, J. H. \& Hawley, J. F. 2002, ApJ, 573, 754

Lovelace, R. V. E., \& Hohlfeld, R. G. 1978, ApJ, 221, 51

Lovelace, R. V. E., Li, H., Colgate, S. A., Nelson, A. F. 1999, ApJ, 513, 805

Luminet, J., -P. 1979, A\&A, 75, 228

Melia, F. 2001, Nature, 413, 25

Melia, F. 2007, in The Galactic Supermassive Black Hole (PUP: New York)
Melia, F. \& Coker, R.F. 1999, ApJ, 511, 750

Melia, F. \& Falcke, H. 2001, ARAA, 39, 309

Melia, F., Prescher, M., Bélanger, G. \& Goldwurm, A. 2007, ApJL, submitted

Misner, C. W., Thorne, K., S., \& Wheeler, J., A. 1973, Gravitation (San Francisco: Freeman)

Poutanen, J., \& Gierliński, M. 2003, MNRAS, 343, 1301

Schödel, R., Ott, R., Genzel, R., Eckart, A., Mouawad, N., \& Alexander, T. 2003, ApJ, 596, 1015

Tagger, M.\& Melia, F. 2006, ApJ, 636, L33 